\title{
A Darwinian view of Behçet's disease
}

\author{
Rhodri Smith ${ }^{1}$, Robert J. Moots ${ }^{1}$, Mariam Murad², Graham R. Wallace ${ }^{2, *}$
}

${ }^{1}$ Department of Rheumatology, Aintree Hospital, Liverpool, UK

${ }^{2}$ Institute of Inflammation and Ageing, College of Medical and Dental Sciences, University of Birmingham, Birmingham, UK

\begin{abstract}
Behçet's disease (BD) is a multisystem inflammatory disorder of unknown etiology, characterized by oral and genital ulceration, with other complications including eye, skin, joint, and central nervous system (CNS) lesions. Diagnosis is based on clinical findings, which may differ between patients. There is a strong genetic basis for BD; however, only a few genes have been associated with the disease across the geographical spread of BD. In this article, we discuss the history and combination of genes involved in this complex disease in relation to the geographical range and present our view that the disease has developed from a Darwinian perspective, with different gene polymorphisms that affect the same biological pathway. Moreover, these mutations individually are protective mechanisms against the disease relevant to each region, which affected both archaic and modern humans.
\end{abstract}

Keywords

genes $•$ Behcet's disease $•$ introgression $\bullet$ neanderthal

\section{Introduction}

The sine qua non of Behçet's disease (BD) is recurrent oral aphthous ulceration, which must be accompanied by two or more of the following major criteria: genital ulceration, uveitis, and skin lesions such as erythema nodosum and a positive pathergy test. A multitude of other clinical features, although not essential for diagnosis, is now recognized to be associated with the disease, such as seronegative arthropathy, increased risk of venous thrombosis, and gastrointestinal inflammation. The cause of BD is unknown, but an autoinflammatory etiology, following infection by an as yet unidentified pathogen, has been suggested. In this scenario, a bacterial or viral infection of mucosa would induce an immune response, leading to cross-reactivity with self-proteins and producing the organ-specific damage, characteristic of $B D{ }^{[1]}$ Although the precise etiology of $B D$ remains mysterious, there is a strong genetic component to the disease, with a range of well-known associations with particular alleles. ${ }^{[2]}$

\section{HLA-B and chromosome 6}

A heritable risk factor for $B D$ was first identified in 1982 when an association with human leukocyte antigen-B5 (HLA-B5) in the human major histocompatibility complex (MHC) was first reported. ${ }^{[3]}$ The HLA-B*5 locus includes the family of HLA-B51 alleles and HLA-B52. In the majority of populations, the greatest heritable risk factor for disease, and indeed for ocular disease severity, is HLA B51. In contrast, HLA-B*52, which differs from HLA-B 51 by only two amino acids in the peptide-binding groove, is not associated with $\mathrm{BD}$ in any population. ${ }^{[4]}$ The geographical distribution of $\mathrm{BD}$, concentrated mainly in countries from the Mediterranean basin to the Far East, led to the synonym of "Silk Road" disease. These regions lie between latitudes $30^{\circ}$ and $45^{\circ}$ north, and it is of interest that the reported prevalence of $\mathrm{BD}$ is associated with latitude and rarely encountered in more northern climes, the Americas, and Australasia. Furthermore, the geographical distribution of HLA-B*51 among healthy subjects roughly corresponds with global disease distribution. ${ }^{[4]}$ This association 
between geographical disease distribution and HLA-B*51 led to the hypothesis that genetic risk factors were propagated by migrant traders operating along the Silk Road some 2000 years ago. ${ }^{[3]}$ However, the new discipline of paleogenetics, involving recent advances in techniques for recovering and analyzing ancient DNA (aDNA), has produced evidence for much earlier acquisition of some HLA alleles, during Paleolithic migrations of modern humans (Homo sapiens) and admixture with archaic human species.

Following the draft sequence of the Neanderthal genome, it was estimated that the Neanderthal contribution represented between $1 \%$ and $4 \%$ of modern Eurasian genomes. ${ }^{[5]}$ Recent estimates of confidently inferred Neanderthal ancestry yield means of $1.38 \%$ and $1.15 \%$ in East Asian and European populations, respectively, but $<0.1 \%$ of the genome in subSaharan African populations. ${ }^{[6]}$ Long stretches of modern Eurasian genomes lacking Neanderthal ancestry suggest that the genetic contribution was initially much larger, estimated at $>3 \%$, but that negative selection has operated to reduce the Neanderthal component now present in modern human genomes. ${ }^{[6]}$

Evidence of another archaic population, genetically distinct from Neanderthals, comes from deoxyribonucleic acid (DNA) extracted from a finger bone found in the Denisova Cave in southern Siberia. ${ }^{[7,8]}$ It is not clear how this population relates to other known hominin species, so the genetically identified population is currently known simply as the "Denisovans". While present-day Melanesian genomes are estimated to contain a Denisovan contribution of $4-6 \%$, the Denisovan contribution to European genomes appears to be negligible. The Denisovan contribution to the genomes of living East Asians and Native Americans is higher, at around $0.2 \%$, but this may be due to admixture with the ancestors of the modern-day inhabitants of Oceania, long after interbreeding with the Denisovans themselves. There is now genetic evidence for three to five instances of interbreeding between hominin populations, including modern humans and Neanderthals, although this is accepted to provide a low-resolution reconstruction of demographic history. ${ }^{[9]}$

Regardless of the imprecision surrounding the events that led to the admixture and incorporation of archaic DNA into modern human genomes, it is now clear that modern humans have received input from archaic genomes and these contributions have variously been both beneficial and deleterious within modern human genomes. Focusing on HLA genes, it seems likely that modern humans acquired the HLA-B*73 allele through admixture with Denisovans. HLA-B* 51 was identified in Neanderthal samples and is found in high frequencies in modern human populations in Eurasia but not in Africa. Simulations of HLA gene introgression strongly supported a Neanderthal origin for HLA-B ${ }^{*} 51$ in modern genomes, among others. ${ }^{[10]}$
The evidence suggests that Neanderthal admixture contributed HLA-B*07, $B^{*} 51, C^{*} 0702$, and $C^{*} 1602$ to modern human genomes. Of these alleles, HLA-B*51 and HLA-C*1602 are associated with BD. Importantly, the frequency of these alleles in modern humans matches the prevalence of BD very closely ${ }^{[10]}$ [Figure 1]. The existence of these HLA alleles in archaic genomes and their preservation in modern genomes, when other Neanderthal genes were subject to negative selection, strongly suggest that these alleles were beneficial in the context of local pathogens encountered by both Neanderthal populations and modern human populations living in similar environments.

Indeed, Neanderthals and their ancestors had been living in Europe and Western Asia for hundreds of thousands of years before modern humans, expanding out of Africa or the Middle East, arrived in that territory. It seems likely that the immune system of Neanderthals had adapted to the local pathogen pool and that this genetic advantage was passed on to modern human populations through interbreeding. The links between admixture, retention of archaic DNA, and disease in human populations, archaic and modern, are likely to be related to genes that were and are protective against infectious diseases. ${ }^{[10]}$ Piga and Mathieu[11] had proposed such a link with Neanderthal admixture and negative selection of HLA-B*5101 and Plasmodium falciparum infection and a positive selection in response to Yersinia pestis to describe the geoepidemiology distribution of BD.

The identification of single nucleotide polymorphisms (SNPs), of Neanderthal origin, that are the risk factors for a range of diseases including systemic lupus erythematosus, biliary cirrhosis, Crohn's disease, smoking behavior, and type 2 diabetes (T2D) further supports a model of the introgression of Neanderthal genes, exerting a positive influence on the survival of early modern humans but leading to increased susceptibility to disease in modern individuals. ${ }^{[6]}$ The current Sars$\mathrm{CoC} 2$ pandemic has supported a role for Neanderthal-derived gene mutation and disease severity. Increased levels of a Neanderthal haplotype of $2{ }^{\prime} 5$ ' oligoadenylate synthase (OAS1) in Europeans have a protective effect in Coronavirus-19 (COVID) infection. ${ }^{[12]}$ By comparison, a Neanderthal core haplotype of a gene cluster on Chr 3 is positively selected in South Asians, particularly Bangladeshis, is associated with a more severe response to COVID. ${ }^{[13]}$

The link between archaic alleles and disease in modern humans is paradoxical; if such alleles were retained due to their protective nature, why are they linked with the disease in current populations? Several possibilities exist: first, until very recently, life expectancy was very short, and in particular, infant mortality very high. Genetic polymorphisms that helped to control pathogens in childhood and adolescence, allowing adulthood to be reached, would be retained-regardless of whether those polymorphisms caused "damage" later in 


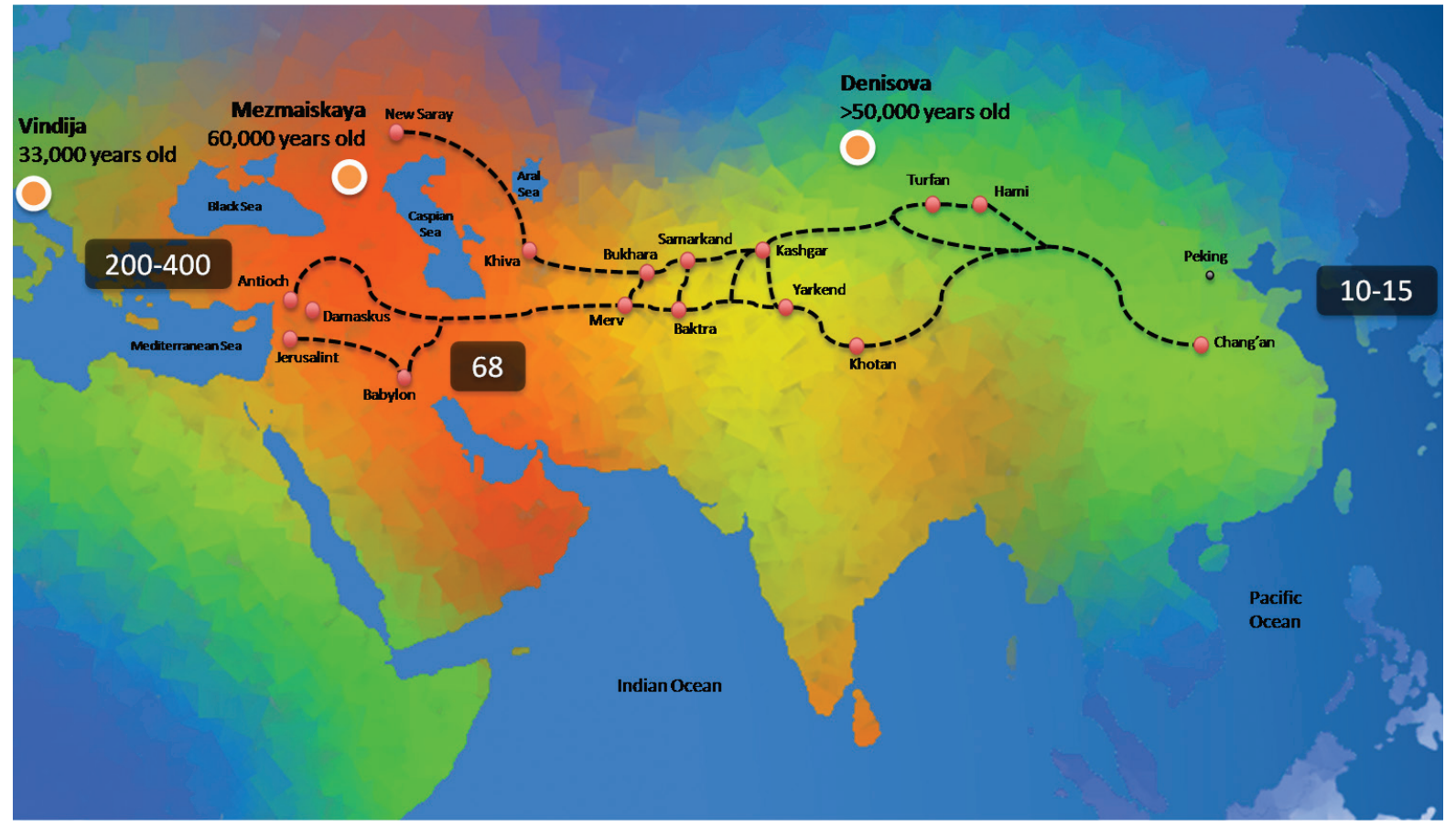

Figure 1: $B D, H L A-B^{\star} 51$, and archaic DNA. The frequency of HLA-B*51 is highest in the Middle East (red-high and green-low) and decreases across the Silk Road (dotted line) to China. The prevalence of BD follows a similar pattern (number/100,000 in boxes). Circles show the sites of bones used in typing archaic genomes. BD, Behçet's disease; HLA-B51, human leukocyte antigen-B51.

life, a concept known as antagonistic pleiotropy. ${ }^{[14]} \mathrm{A}$ second possibility is that genes that were protective in the Paleolithic have a very different effect today, where diet and lifestyle are much changed from those of our ancestors. For instance, modern diseases such as Crohn's and T2D have been linked to changes in diet including increased sugar intake, which leads to changes in the gut microbiome that play an important role in changing profiles of disease. ${ }^{[15]}$ Finally, combinations of gene variants maintained by the mechanisms described above are responsible for many modern diseases. This Darwinian concept of many small, mainly positive, changes accumulating over time is supported by the large number of SNPs identified in many complex diseases.

\section{HLA-B*51 and Behcet's disease}

While an obvious interpretation of the role of HLA-B*51 in $B D$ is the presentation of antigen to $C D 8^{\text {tve }} T$ cells, the association with HLA-B*51 could be due to its interaction with killer cell immunoglobulin-like receptor (KIR) genes, which are also highly polymorphic and diversely expressed on natural killer (NK) cells, which for inhibitory receptors is under genetic control, whereas activatory receptors are under environmental control. NK cell function is therefore strictly controlled by the expression of the inhibitory receptors. Alterations in either allele expression of inhibitory KIR or environmental-induced changes in activatory KIR could influence disease prevalence. ${ }^{[16]}$ While HLA-B loci have been identified in multiple genetic studies as the strongest association, recent deep sequencing of the HLA loci in BD patients with imputation studies based on the 1000 Genomes Project was performed in two different independent BD cohorts. The robust association with HLA-B*51 was explained by a variant between $B^{\star} 51$ and $\mathrm{MHC}$ class 1-related A (MICA; rs116799036), which was independent of $B^{*} 51$. Additional independent associations in psoriasis susceptibility locus (PSORS1), HLA-f-AS1, and HLA-Cw*1602 were also identified and replicated. ${ }^{[17]}$ However, the independence of rs 116799036 was not supported in the analysis of a larger cohort of patients that strongly backed HLA-B as the primary gene association on chromosome 6 . Importantly, the amino acids relevant to the HLA-B*51 association were identified and included HLA-B* 67 , which is one of the two amino acid differences between $B^{*} 51$ and $B^{*} 52$. The latter is protective in Middle Eastern patients with BD. ${ }^{\left[{ }^{18]}\right]}$ In support of the concept that HLA-B has been the major risk for $B D$, imputation studies identified SNP in endoplasmic reticulum-expressed aminopeptidase (ERAP1) (rs17482078). There was evidence of epistasis between ERAP1 and $H L A-B^{*} 51$. ERAP1 is a protein involved in trimming peptides to fit MHC class I molecules; mutations conferred risk for BD in HLA-B* $51^{\text {tve }}$ individuals, with homozygosity of ERAP1 rs17482070 giving an OR of 3.78. ${ }^{[19]} \mathrm{A}$ haplotype, based on ERAP-1 sequencing, designated Hap10 with five non-ancestral amino acids was reported to be associated with BD. ${ }^{[20]}$ Individual variants encoded by this haplotype rs2287987, rs10050860, and rs17482078 were previously reported by studies in various ethnic groups to be 
associated with BD. ${ }^{[21,22]}$ Such variants influence the binding of low-affinity peptidomes to HLA-B*5101, which may be relevant in BD. Specifically, 2 subpeptidomes were identified, whereby only peptides in the subpeptidome with Ala at position 2 are extensively destroyed, except when their position 1 residues are ERAP-1 resistant. ${ }^{[23,24]}$ Recently, KIR3DL1 allele-level analysis revealed the KIR3DL1LOW/KIR3DS1 functional genotype to be implicated in disease pathogenesis and KIR3DL $1^{\text {HIGH}} / K I R 3 D L 1^{\text {NULL }}$ to be protective. ${ }^{[25]}$ These data suggest a complex mechanism by which HLA-B*5101 is involved in $\mathrm{BD}$ and more functional studies are required to elucidate the process.

\section{Other loci on chromosome 6}

Tumor necrosis factor (TNF) is a pro-inflammatory cytokine whose serum levels are raised in patients with BD and inhibition of TNF by various biologic drugs is effective in controlling the disease. The gene for TNF is on human chromosome 6 . Many SNPs in TNF have been analyzed since the -1031 promoter allele was associated with BD in UK patients. ${ }^{[26]}$ Subsequently, $-1031 \mathrm{C}$ has been linked with the disease in Turkish and Tunisian with BD. ${ }^{[27,28]}$ Similarly, $-308 \mathrm{~A} / \mathrm{G}$ in TNF has been associated with disease in Iranian Azeri Turks but not in Palestinian patients. ${ }^{[4,29]}$ In Korean patients, none of the tested TNF SNP were associated with the disease. ${ }^{[30]}$ Therefore, SNPs in this important proinflammatory cytokine are associated with $\mathrm{BD}$ in many populations, but which polymorphism would appear to differ in different geographical cohorts.

\section{Non-chromosome 6 associations}

\section{(a) Lymphocyte Signaling}

Genome-wide analysis studies (GWAS) in a cohort of 379 Korean patients with Behçet's and 800 controls have found a significant association with the GTPase of immunity associated protein family (GIMAP) gene cluster on chromosome 7q36.1. Genome-wide significance was found using a dominant model of inheritance for 5 SNPs within the GIMAP cluster (rs1608157, rs1522596, rs10266069, rs10256482, and rs2286900) with subsequent fine-mapping efforts finding an association with 10 additional markers although these were distributed across 3 blocks of linkage disequilibrium (LD) within this $\sim 200 \mathrm{kbp}$ region. Replication of the signal was achieved for SNPs mapping to the GIMAP2 and GIMAP4 regions, but not GIMAP1, in a Japanese cohort. Additionally, functional data of $31 \mathrm{BD}$ patients showed significantly reduced expression of GIMAP1, GIMAP4 (both in CD4 T cells), and GIMAP2 (in CD8 T cells) when compared to controls $(P<0.05)$. Further to this, using a luciferase reporter assay they found that a construct having the risk allele $(C)$ within rs1608157 locus (the most significant GWAS SNP) had reduced luciferase activity compared to the $G$ allele implying that the polymorphism negatively affected GIMAP4 promoter activity. However, GIMAP SNPs were not validated in the UK or European patients with BD. ${ }^{[31,32]}$

Several other genes linked to the severity of particular manifestations of BD, associated with $T$ cell signaling, have been reported and many of these have been validated in multiple studies. Certain gene polymorphisms associated with other autoimmune diseases, including protein tyrosine phosphatase non-receptor type 22 (PTPN22) and cytotoxic T-lymphocyte antigen 4 (CTLA-4), were shown to have a negative association-results that support the concept that $B D$ is an autoinflammatory rather than an autoimmune disease. ${ }^{[33]}$ PTPN22 R620W polymorphism is most prevalent in northern Europe and protective against Mycobacteria tuberculosis but induces susceptibility to Mycobacteria leprae, which was more common in the Middle East, where PTPN22 R620W is very rare. PTPN22 R620W was inversely associated with $B D$ in patients particularly in a UK cohort. ${ }^{[34]}$ This association was seen neither in patients from the Middle East and Southern Europe, where the prevalence of the polymorphism is much lower, nor in Chinese patients with BD, where the polymorphism does not exist. ${ }^{[35-37]}$ PTPN22 encodes a protein Lyp that binds to Csk, an inhibitory kinase, and together disrupt T cell signaling through dephosphorylation of Lck. The R620W SNP disrupts this initial interaction. ${ }^{[38]}$ In myeloid cells, PTPN22 positively increases type 1 interferon production, and dependent viral infection and gut homeostasis. ${ }^{[39]}$ In mice, carriage of $620 \mathrm{~W}$ led to increased autoreactive $\mathrm{T}$ cell clones in new bone-marrow emigrants. ${ }^{[40]}$ Inhibition of PTPN22 activity restored central B cell tolerance. ${ }^{[41]}$ Superresolution microscopy showed that while wild type PTPN22 forms large clusters in unstimulated $\mathrm{T}$ cells and dissociates on stimulation by LFA-1, on ICAM-1 monolayers, enabling association with Lck and ZAP70 at the leading edge. By comparison, PTPN22 620W was not retained at the leading edge resulting in increased LFA-1 clustering and integrin-mediated adhesion. ${ }^{[42]}$ PTPN22 is also highly expressed in neutrophils. R620W enhanced neutrophil migration and increased $\mathrm{Ca}+$ release, in humans, and is a critical regulator of FcR activation and interaction with immune complexes by neutrophils in mice. ${ }^{[43-45]}$

For CTLA4 polymorphisms, the situation is even more complex. Early studies on Turkish and Tunisian patients with BD found associations with certain manifestations of the disease. ${ }^{[46,47]}$ However, studies on the UK, Middle Eastern, and Chinese patients showed no association. ${ }^{[48-50]}$ Meta-analysis of eight studies including those above showed no association with $B D .{ }^{[51]}$ However, whether certain features of $B D$ are linked to CTLA4 polymorphisms or they are associated with other combinations of genes will need larger studies.

A signal transducer and activator of transcription 4 (STAT4) association are supported by a study on Han Chinese patients with BD, with SNP rs7574070 and additional SNPs rs7572482 and rs897200, all associated although in strong 
LD. In addition, Hou et al. showed that BD patients' homozygous for the rs 897200 risk allele had increased expression of the STAT4 mRNA and protein, as well as IL-17, which is regulated by the gene. ${ }^{[52]}$ Furthermore, ENCODE data reveal that the rs897200 polymorphism determines the formation of a transcription factor binding site, and the functional studies by the authors indicate that this SNP is likely to be an expression of quantitative trait locus (eQTL) of STAT4. Association of STAT4 was further reported in patients with BD from Turkey and Iran. ${ }^{[24,53]}$ Although a STAT4 gene association (rs7574865) has been reported in a number of autoimmune diseases including RA and systemic lupus erythematosus, variants associated with $B D$ are in low $L D$, with this particular marker implying that such associations are independent of each other and, therefore, may confer different mechanisms of pathogenesis. ${ }^{[24]}$

Therefore, several polymorphisms in genes involved with $\mathrm{T}$ cell signaling are reported in BD but not in all populations. However, that many genes signaling through different, though interconnected, pathways in different geographical populations influence the same outcome that supports a Darwinian concept of BD [Figure 2].

\section{(b) Interleukin-10 (IL10)/IL12R/IL23R}

GWAS identified polymorphisms in the $I L 10$ and IL23R/ IL12RB2 genes, which may help to explain the inflammatory response in $\mathrm{BD} \cdot{ }^{[54,55]}$ Both $I L 10$ as an anti-inflammatory molecule and IL23R as a member of the pro-inflammatory IL-17 pathway have been identified as associated with BD

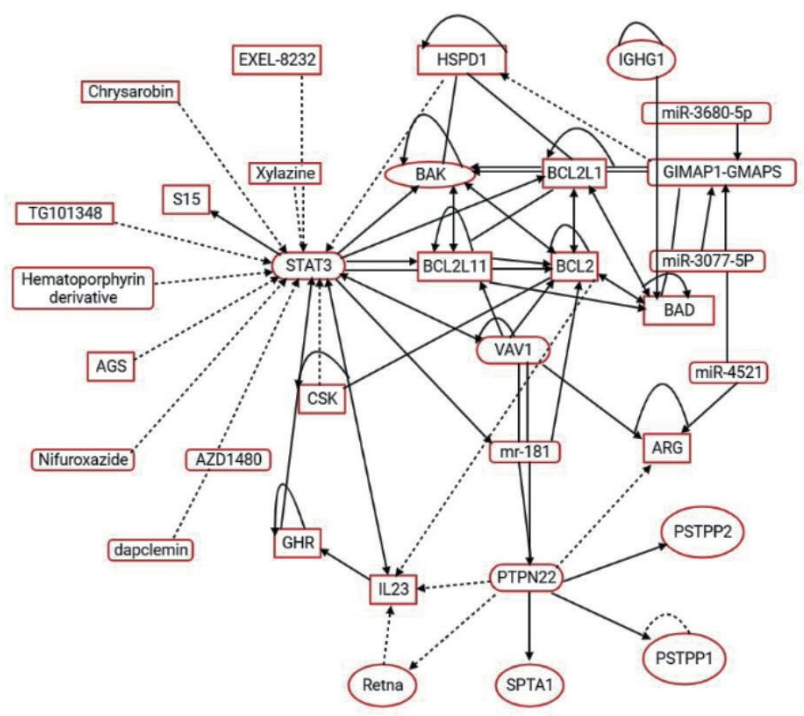

Figure 2: GIMAP, and PTPN22, identified with $B D$ in different geo graphic populations are linked to many similar molecules that are involved in lymphocyte activation such as STAT3 (signal transducer and activator of transcription 3). Image in Biorender. in candidate gene studies and, therefore, their detection in GWAS is intriguing. ${ }^{[56,57]}$ The interleukin genes IL10's and IL23R's roles within BD pathogenesis have been elucidated from the above-described independent but concurrent Turkish and Japanese GWAS. Genotyping identified an association with the IL10 intronic rs 1518111 variant in a combined cohort, which correlated with IL-10 production by blood monocytes, implicating low IL-10 as a risk factor of BD. ${ }^{[5]}$ A second intronic SNP rs 1554286 was initially associated with $\mathrm{BD}$ in the Japanese study; however, further investigation and LD analysis fine mapped the association to the $I L 10$ promoter region with variants rs 1800872 and rs 1800871 having a stronger association. A subsequent meta-analysis of the data with additional Korean samples and Turkish GWAS data identified rs1800871 as the most significant risk marker. Replication of the IL-10 association has since been achieved in other geographical cohorts of patients with BD. ${ }^{[58]}$

Within a $\sim 50 \mathrm{kbp}$ intergenic region between the IL23R and IL12RB2 genes on chromosome 1, SNPs rs12119179, rs1495695, rs17375018, and rs924080 have all been associated with BD from GWAS. SNP rs12119179 was initially associated with the Japanese cohort. ${ }^{[59]}$ Fine mapping showed a stronger association with rs1495965 which in meta-analysis of a Korean cohort. ${ }^{[6]}$ No association with this region was detected in the Turkish GWAS alone although SNP rs924080 was significantly associated in their combined cohort. ${ }^{[5]}$ Recent functional analysis in PBMCs has further demonstrated that the rs924080 risk allele increases expression of IL6 and augments the expression capacity of both IL23R and TNF- $\alpha$ when stimulated with lipopolysaccharide, leading the authors to suggest that the risk allele underpins an inappropriately increased inflammatory response to bacterial pathogens that ultimately increases the risk of BD onset. ${ }^{[61]}$ In addition to this, an earlier study investigating the IL23 signaling pathway genes had shown evidence of BD association with IL23R SNPs rs17375018, rs11209032, and rs924080 in Chinese cohorts, although there has been no GWAS association with this region within Chinese Han to date. ${ }^{[62,63]}$

\section{(c) Pathogen Recognition}

Pathogens are recognized by a family of molecules, the best known of which are Toll-like receptors (TLR). Non-synonymous variants identified by deep-sequencing of 10 identified genes (GWAS), and 11 identified by association with innate immunity, Table 1 showed TLR4 was associated with BD, while rare nucleotide-binding oligomerization domain-2 (NOD2) variants were nominally significant. TLR4 variants that are risk factors in Crohn's are protective in BD. ${ }^{[64]}$ TLR expression in buccal lesions from BD patients showed an increase in positive cells, but this was no different from other inflammatory conditions tested. Polymorphisms in TLR2 and TLR4 showed no association with $\mathrm{BD}$; however, a polymorphism in 


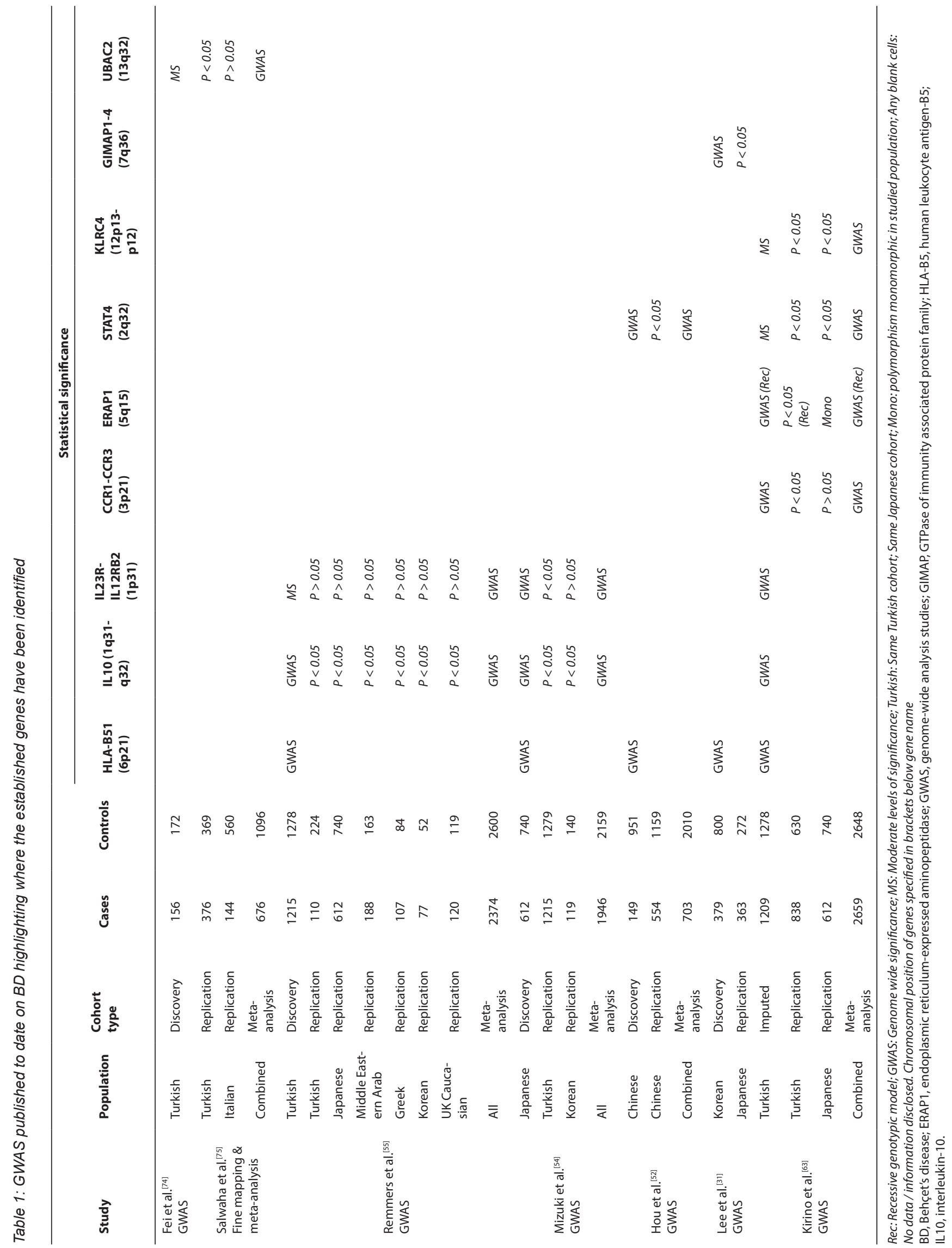


the downstream TLR signaling molecule, TIRAP, that leads to gain of function is associated with BD. ${ }^{[65]}$ SNPs in TLR2 rs2289318 and rs3804099 were associated with ocular disease in Han Chinese patients with BD. Both polymorphisms were linked to altered TLR2 mRNA expression in peripheral blood mononuclear cells stimulated with peptidoglycan. ${ }^{[6]}$ NOD2 is an intracellular recognition molecule that recognizes muramyl dipeptide present in some bacteria. SNPs in NOD2 associated with Crohn's disease were not associated with $\mathrm{BD}$ in British, Turkish, or Arab patients in initial studies, with one SNP inversely associated with the disease. ${ }^{[67]}$ A potential protective association was reported in a second study with SNP to be protective in Caucasian patients with BD. ${ }^{[68]}$ In a recent study of a Spanish cohort, a single rare SNP was associated with the protection of patients with BD. ${ }^{[69]}$

FUT2 encodes an $\alpha-(1,2)$ fucosyltransferase that regulates the secretion of the $\mathrm{H}$ antigen (precursor of the human $\mathrm{ABO}$ blood group antigens) in body fluids and intestinal mucosa. About $80 \%$ of people have the secretor phenotype, which is determined by the presence of at least one functional FUT2 allele. FUT2 is expressed in the intestinal epithelial cells and fucosylated proteins are shed into the lumen Fucose, which is metabolized by certain gut bacteria that induce short-chain fatty acids such as butyrate and downregulating virulence genes in other bacterial spp. FUT2 is increased in epithelial cells via TLR signaling. ${ }^{[70]}$ Five coding SNPs were identified in Iranian patients with BD. ${ }^{[71]}$ Meta-analysis of Iranian and Turkish GWAS data supported the initial report that rs601338 allele is associated with disease in Turks and Iranians patients with BD and rs1047781 allele is associated with disease in Japanese patients. ${ }^{[72]}$ These non-secretor genotypes have been associated with increased predisposition or resistance to different infectious agents such as norovirus but are not associated with the gut microbiome composition. ${ }^{[3]}$

\section{Conclusion}

The strongest association with $\mathrm{BD}$ is $H L A-B{ }^{*} 51$ on chromosome 6 , a link that has been confirmed in several studies in different geographical groups. HLA-B*51 is derived from Neanderthal admixture and is likely to have been maintained in modern humans as part of a protective mechanism against a disease that affected both archaic and modern humans. Beyond chromosome 6, SNPs in genes involved in lymphocyte signaling and function, along with ubiquitination and pathogen recognition, which may be protective in evolutionary time but when brought together represent further evidence supporting a Darwinian view of BD. The identification of different polymorphisms in different geographical cohorts of patients with $\mathrm{BD}$ supports the accumulation of small mutations that, when combined with $H L A-B^{*} 51$, lead to the disease. However, it is now becoming clear that these different, positively selected SNPs are, in many cases, affecting the same biological pathways. An excellent, comprehensive genetic and epigenetic analysis identified a heritability of $60 \%$ in $\mathrm{BD}$ incorporating mutations in genes associated with the immune response, including interferon-gamma and IL-12 production, regulation of lymphocyte-mediated immunity cytokine-cytokine receptor interaction, and regulation of innate immune response. Genetic cumulative risk scores supported the known prevalence of BD globally. ${ }^{[7]}$ These new insights cast light on the biochemical pathway involved in BD, beyond links with individual genes. This emerging understanding of the evolutionary, genetic, and biochemical basis of $\mathrm{BD}$ may explain much of the variation in BD prevalence with geography and may even suggest new directions for therapeutic research. In conclusion, protective genes inherited from archaic humans in the distant past, interacting with other polymorphisms in modern humans, may explain the strong link that we observe between $H L A-B$ and BD.

Conflict of Interest

Robert J. Moots is the Co-Editor-in-Chief of the journal. The article was subject to the journal's standard procedures, with peer review handled independently of this editor and his research groups.

References

[1] Saadoun D, Wechsler B. Behcet's Disease. Orphanet J Rare Dis, 2012;7(1):1-6.

[2] Gul A. Genetics of Behcet's Disease: Lessons Learned from Genomewide Association Studies. Curr Opin Rheumatol, 2014;26(1):56-63.

[3] Ohno S, Ohguchi M, Hirose S, et al. Close Association of HLA-Bw51 with Behcet's Disease. Arch Ophthalmol, 1982;100(9):1455-1458.

[4] Verity DH, Marr JE, Ohno S, et al. Behcet's Disease, the Silk Road and HLA-B51: Historical and Geographical Perspectives. Tissue Antigens, 1999;54(3):213-220.

[5] Green RE, Krause J, Briggs AW, et al. A Draft Sequence of the Neandertal Genome. Science, 2010;328(5979):710-722.

[6] Sankararaman S, Mallick S, Dannemann M, et al. The Genomic Landscape of Neanderthal Ancestry in Present-day Humans. Nature, 2014;507(7492):354-357.

[7] Krause J, Fu Q, Good JM, et al. The Complete Mitochondrial DNA 
Genome of an Unknown Hominin from Southern Siberia. Nature, 2010;464(7290):894-897.

[8] Reich D, Green RE, Kircher M, et al. Genetic History of an Archaic Hominin Group from Denisova Cave in Siberia. Nature, 2010;468(7327):1053-1060.

[9] Prufer K, Racimo F, Patterson N, et al. The Complete Genome Sequence of a Neanderthal from the Altai Mountains. Nature, 2014;505(7481):43-49.

[10] Abi-Rached L, Jobin MJ, Kulkarni S, et al. The Shaping of Modern Human Immune Systems by Multiregional Admixture with Archaic Humans. Science, 2011;334(6052):89-94.

[11] Piga M, Mathieu A. The Origin of Behcet's Disease Geoepidemiology: Possible Role of a Dual Microbial-driven Genetic Selection. Clin Exp Rheumatol, 2014;32(S84):S123-S129.

[12] Zeberg H, Pääbo S. A Genomic Region Associated with Protection Against Severe COVID-19 is Inherited from Neandertals. Proc Natl Acad Sci U S A, 2021;118(9):e2026309118. doi: 10.1073/ pnas.2026309118.

[13] Zhou S, Butler-Laporte G, Nakanishi T, et al. A Neanderthal OAS1 Isoform Protects Individuals of European Ancestry Against COVID-19 Susceptibility and Severity. Nat Med, 2021;27(4): 659-667. doi: 10.1038/s41591-021-01281-1.

[14] Williams GC. Pleiotropy, Natural Selection, and the Evolution of Senescence. Evolution (BioOne), 1957;11:398-411.

[15] Pfeffer-Gik T, Levine A. Dietary Clues to the Pathogenesis of Crohn's Disease. Dig Dis, 2014;32(4):389-394.

[16] Horowitz A, Strauss-Albee DM, Leipold M, et al. Genetic and Environmental Determinants of Human NK Cell Diversity Revealed by Mass Cytometry. Sci TransI Med, 2013;5(208):208ra145.

[17] Hughes T, Coit P, Adler A, et al. Identification of Multiple Independent Susceptibility Loci in the HLA Region in Behcet's Disease. Nat Genet, 2013;45(3):319-324.

[18] Ombrello MJ, Kirino Y, de Bakker PI, et al. Behcet Diseaseassociated MHC Class I Residues Implicate Antigen Binding and Regulation of Cell-mediated Cytotoxicity. Proc Natl Acad Sci U S A, 2014;111(24):8867-8872.

[19] Kirino Y, Bertsias G, Ishigatsubo Y, et al. Genome-wide Association Analysis Identifies New Susceptibility Loci for Behcet's Disease and Epistasis Between HLA-B*51 and ERAP1. Nat Genet, 2013;45(2):202-207.

[20] Takeuchi M, Ombrello MJ, Kirino Y, et al. A Single Endoplasmic Reticulum Aminopeptidase-1 Protein Allotype is a Strong Risk Factor for Behçet's Disease in HLA-B ${ }^{\star} 51$ Carriers. Ann Rheum Dis, 2016;75(12):2208-2211.

[21] Zhang L, Yu H, Zheng M, et al. Association of ERAP1 Gene Polymorphisms with Behçet's Disease in Han Chinese. Invest Ophthalmol Vis Sci, 2015;56(10):6029-6035.

[22] Padula MC, Leccese P, Pellizzieri E, et al. Distribution of rs17482078 and rs27044 ERAP1 Polymorphisms in a Group of Italian Behçet's Syndrome Patients: A Preliminary Case-control Study. Intern Emerg Med, 2019;14(5):713-718.

[23] Guasp P, Barnea E, González-Escribano MF, et al. The Behçet's Disease-associated Variant of the Aminopeptidase ERAP1 Shapes a Low-affinity HLA-B*51 Peptidome by Differential Subpeptidome Processing. J Biol Chem, 2017;292(23):9680-9689.
[24] Guasp P, Alvarez-Navarro C, Gomez-Molina P, et al. The Peptidome of Behçet's Disease-associated HLA-B*51:01 Includes Two Subpeptidomes Differentially Shaped by Endoplasmic Reticulum Aminopeptidase 1. Arthritis Rheumatol, 2016;68(2):505-515.

[25] Petrushkin H, Norman PJ, Lougee E, et al. KIR3DL1/S1 Allotypes Contribute Differentially to the Development of Behçet Disease. J Immunol, 2019;203(6):1629-1635.

[26] Ahmad T, Wallace GR, James T, et al. Mapping the HLA Association in Behcet's Disease: A Role for Tumor Necrosis Factor Polymorphisms? Arthritis Rheum, 2003;48(3):807-813.

[27] Akman A, Sallakci N, Coskun M, et al. TNF-a Gene 1031 T/C Polymorphism in Turkish Patients with Behcet's Disease. $\mathrm{Br} J$ Dermatol, 2006;155(2):350-356.

[28] Kamoun M, Chelbi H, Houman, MH, et al. Tumor Necrosis Factor Gene Polymorphisms in Tunisian Patients with Behcet's Disease. Hum Immunol, 2007;68(3):201-205.

[29] Bonyadi M, Jahanafrooz Z, Esmaeili M, et al. TNF-a Gene Polymorphisms in Iranian Azeri Turkish Patients with Behcet's Disease. Rheumatol Int, 2009;30(2):285-289.

[30] Lee EB, Kim JY, Lee YJ, et al. TNF and TNF Receptor Polymorphisms in Korean Behcet's Disease Patients. Hum Immunol, 2003;64(6):614-620.

[31] Lee YJ, Horie Y, Wallace GR, et al. Genome-wide Association Study Identifies GIMAP as a Novel Susceptibility Locus for Behcet's Disease. Ann Rheum Dis, 2013;72(9):1510-1516.

[32] Ortiz-Fernández L, Conde-Jaldón M, García-Lozano JR, et al. GIMAP and Behçet Disease: No Association in the European Population. Ann Rheum Dis, 2014;73(7):1433-1434.

[33] Direskeneli H. Autoimmunity vs Autoinflammation in Behcet's Disease: Do We Oversimplify a Complex Disorder. Rheumatology (Oxford), 2006;45(12):1461-1465.

[34] Baranathan V, Stanford MR, Vaughan RW, et al. The Association of the PTPN22 620W Polymorphism with Behcet's Disease. Ann Rheum Dis, 2007;66(11):1531-1533.

[35] Sahin N, Bicakcigil M, Atagunduz P, et al. PTPN22 Gene Polymorphism in Behcet's Disease. Tissue Antigens, 2007;70(5):432-434. [36] Ortiz-Fernández L, Montes-Cano MA, García-Lozano JR, et al. PTPN22 is not Associated with Behçet's Disease. Study Spanning the Complete Gene Region in the Spanish Population and Metaanalysis of the Functional Variant R620W. Clin Exp Rheumatol, 2016; 34(S102):S41-S45.

[37] Zhang Q, Hou S, Jiang Z, et al. No Association of PTPN22 Polymorphisms with Susceptibility to Ocular Behcet's Disease in Two Chinese Han Populations. PLoS One, 2012;7(3):e31230.

[38] Brownlie RJ, Zamoyska R, Salmond RJ. Regulation of Autoimmune and Anti-tumour T-cell Responses by PTPN22. Immunology, 2018;154(3):377-382.

[39] Wang Y, Shaked I, Stanford SM, et al. The Autoimmunity-associated Gene PTPN22 Potentiates Toll-like Receptor-driven, Type 1 Interferon-dependent Immunity. Immunity, 2013;39(1):111-122.

[40] Sanchez-Blanco C, Clarke F, Cornish GH, et al. Protein Tyrosine Phosphatase PTPN22 Regulates LFA-1 Dependent Th1 Responses. J Autoimmun, 2018;94:45-55.

[41] Schickel JN, Kuhny M, Baldo A, et al. PTPN22 Inhibition Resets Defective Human Central B Cell Tolerance. Sci Immunol, 
2016;1(1):aaf7153. 2.

[42] Burn GL, Cornish GH, Potrzebowska K, et al. Superresolution Imaging of the Cytoplasmic Phosphatase PTPN22 Links Integrin-mediated T Cell Adhesion with Autoimmunity. Sci Signal, 2016; 9(448):ra99.

[43] Bayley R, Kite KA, McGettrick HM, et al. The Autoimmuneassociated Genetic Variant PTPN22 R620W Enhances Neutrophil Activation and Function in Patients with Rheumatoid Arthritis and Healthy Individuals. Ann Rheum Dis, 2015;74(8):1588-1595.

[44] Vermeren S, Miles K, Chu JY, et al. PTPN22 Is a Critical Regulator of Fcy Receptor-Mediated Neutrophil Activation. J Immunol, 2016;197(12):4771-4779.

[45] Clarke F, Purvis HA, Sanchez-Blanco C, et al. The Protein Tyrosine Phosphatase PTPN22 Negatively Regulates Presentation of Immune Complex Derived Antigens. Sci Rep, 2018;8(1):12692.

[46] Ben Dhifallah I, Chelbi H, Braham A, et al. CTLA-4 +49A/G Polymorphism is Associated with Behcet's Disease in a Tunisian Population. Tissue Antigens,2009;73(3):213-217.

[47] Gunesacar R, Erken ER, Bozkurt B, et al. Analysis of CD28 and CTLA-4 Gene Polymorphisms in Turkish Patients with Behcet's Disease. Int J Immunogenet, 2007;34(1):45-49.

[48] Bye L, Modi N, Stanford MR, et al. CTLA-4 Polymorphisms are not Associated with Ocular Inflammatory Disease. Tissue Antigens, 2008;72(1):49-53.

[49] Sallakci N, Bacanli A, Coskun M, et al. CTLA-4 Gene 49A/G Polymorphism in Turkish Patients with Behcet's Disease. Clin Exp Dermatol, 2005;30(5):546-550.

[50] Du L, Yang P, Hou S, et al. No Association of CTLA-4 Polymorphisms with Susceptibility to Behcet Disease. Br J Ophthalmol, 2009;93(10):1378-1381.

[51] Lee YH, Song GG. CTLA-4 Polymorphisms and Susceptibility to Behcet's Disease: A Meta-analysis. Mol Biol Rep, 2012;39(9): 9041-9045.

[52] Hou S, Yang Z, Du L, et al. Identification of a Susceptibility Locus in STAT4 for Behcet's Disease in Han Chinese in a Genome-wide Association Study. Arthritis Rheum, 2012;64(12):4104-4113.

[53] Sousa I, Shahram F, Francisco D, et al. Brief Report: Association of CCR1, KLRC4, IL12A-AS1, STAT4, and ERAP1 with Behçet's Disease in Iranians. Arthritis Rheumatol, 2015;67(10):2742-2748.

[54] Mizuki N, Meguro A, Ota M, et al. Genome-wide Association Studies Identify IL23R-IL12RB2 and IL10 as Behcet's Disease Susceptibility Loci. Nat Genet,2010;42(8):703-706.

[55] Remmers EF, Cosan F, Kirino Y, et al. Genome-wide Association Study Identifies Variants in the MHC Class I, IL10, and IL23RIL12RB2 Regions Associated with Behcet's Disease. Nat Genet, 2010;42(8):698-702.

[56] Wallace GR, Kondeatis E, Vaughan RW, et al. IL-10 Genotype Analysis in Patients with Behcet's Disease. Hum Immunol, 2007;68(2):122-127.

[57] Jiang Z, Yang P, Hou S, et al. IL-23R Gene Confers Susceptibility to Behcet's Disease in a Chinese Han Population. Ann Rheum Dis, 2010;69(7):1325-1328.

[58] Jung JH, Song GG, Lee YH. Meta-Analysis of Associations Between Interleukin-10 Polymorphisms and Susceptibility to Vasculitis. Immunol Invest, 2015;44(6):553-565.
[59] Kang EH, Kim S, Park MY, et al. Behçet's Disease Risk Association Fine-mapped on the IL23R-IL12RB2 Intergenic Region in Koreans. Arthritis Res Ther, 2017;19(1):227-232.

[60] Cavus F, Ulusoy C, Orcen A, et al. Increased IL-23 Receptor, TNF- $\alpha$ and IL-6 Expression in Individuals with the IL23R-IL12RB2 Locus Polymorphism. Immunol Lett, 2014;160(1):96-98.

[61] Jiang Z, Yang P, Hou S, et al. IL-23R Gene Confers Susceptibility to Behcet's Disease in a Chinese Han Population. Ann Rheum Dis, 2010;69(7):1325-1328.

[62] Yu H, Zheng M, Zhang L, et al. Identification of Susceptibility SNPs in IL10 and IL23R-IL12RB2 for Behçet's Disease in Han Chinese. J Allergy Clin Immunol, 2017;139(2):621-627.

[63] Kirino $Y$, Zhou Q, Ishigatsubo Y, et al. Targeted Resequencing Implicates the Familial Mediterranean Fever Gene MEFV and the Toll-like Receptor 4 Gene TLR4 in Behçet Disease. Proc Natl Acad Sci U S A, 2013;110(20):8134-8139.

[64] Durrani O, Banahan K, Sheedy FJ, et al. TIRAP Ser180Leu Polymorphism is Associated with Behcet's Disease. Rheumatology (Oxford), 2011;50(10):1760-1765.

[65] Fang J, Hu R, Hou S, et al. Association of TLR2 Gene Polymorphisms with Ocular Behcet's Disease in a Chinese Han Population. Invest Ophthalmol Vis Sci, 2013;54(13):8384-8392.

[66] Ahmad T, Zhang L, Gogus F, et al. CARD15 Polymorphisms in Behcet's Disease. Scand J Rheumatol, 2005;34(3):233-237.

[67] Kappen JH, Wallace GR, Stolk L, et al. Low Prevalence of NOD2 SNPs in Behcet's Disease Suggests Protective Association in Caucasians. Rheumatology (Oxford), 2009;48(11): 1375-1357.

[68] Burillo-Sanz S, Montes-Cano MA, García-Lozano JR, et al. Behçet's Disease and Genetic Interactions Between HLA-B*51 and Variants in Genes of Autoinflammatory Syndromes. Sci Rep, 2019; 9(1):2777.

[69] Pickard JM, Maurice CF, Kinnebrew MA, et al. Rapid Fucosylation of Intestinal Epithelium Sustains Host-commensal Symbiosis in Sickness. Nature, 2014;514(7524):638-641.

[70] Xavier JM, Shahram F, Sousa I, et al. FUT2: Filling the Gap Between Genes and Environment in Behçet's Disease? Ann Rheum Dis, 2015;74(3):618-624.

[71] Takeuchi M, Mizuki N, Meguro A, et al. Dense Genotyping of Immune-related Loci Implicates Host Responses to Microbial Exposure in Behcet's Disease Susceptibility. Nat Genet, 2017;49(3): 438-443.

[72] Nordgren J, Svensson L. Genetic Susceptibility to Human Norovirus Infection: An Update. Viruses, 2019;11(3):226.

[73] Ortiz-Fernández L, Sawalha AH. Genetics of Behçet's Disease: Functional Genetic Analysis and Estimating Disease Heritability. Front Med (Lausanne), 2021;8:625710. doi: 10.3389/ fmed.2021.625710

[74] Fei Y, Webb R, Cobb BL, et al. Identification of Novel Genetic Susceptibility Loci for Behcet's Disease Using a Genome-wide Association Study. Arthritis Res Ther, 2009;11(3):1-7.

[75] Sawalha AH, Hughes T, Nadig A, Yılmaz V, Aksu K, Keser G, et al. A putative functional variant within the UBAC2 gene is associated with increased risk of Behcet's disease. Arthritis Rheum, 2011; 63(11):3607-12. doi: 10.1002/art.30604. 